\title{
Gamma-Ray Pulsar Emission Models
}

\author{
Alice K. Harding \\ Code 661, NASA/Goddard Space Flight Center, Greenbelt, MD 20771
}

\begin{abstract}
With the increased sensitivity of gamma-ray detectors on the Compton Gamma-Ray Observatory (CGRO) the number of presently known gamma-ray pulsars has grown. The new detections are beginning to provide clues to the origin of the high-energy radiation in the form of emerging patterns and correlations among observed quantities such as gamma-ray efficiency and spectral index vs. age. But there are still many questions about the location of the emission and its relation to the radio, optical and X-ray pulses. This paper will review models for gamma-ray emission from pulsars and will examine how well the detailed predictions of these models account for the existing observations.
\end{abstract}

\section{Introduction}

As the number of $\gamma$-ray pulsars has grown from two to eight over the five years since the launch of CGRO, the amount of new data on these objects has provided many puzzles, some clues, but few definitive answers. Rather, the models for $\gamma$-ray pulsars that were proposed and investigated in the pre-CGRO era have been evolving in light of the new results. There are currently two types of $\gamma$-ray pulsar models being studied in detail. Polar cap models assume that particles are accelerated along open field lines near the neutron star by parallel electric fields and induce pair cascades by either curvature radiation (Daugherty \& Harding 1982, Usov \& Melrose 1995) or inverse-Compton radiation (Dermer \& Sturner 1994). Outer gap models assume that primary particles are accelerated in vacuum gaps in the outer magnetosphere and induce pair cascades through $\gamma$ $\gamma$ pair production (Cheng, Ho \& Ruderman 1986; Romani \& Yadigaroglu 199.5). In addition, there are ideas for generating pulsed $\gamma$-ray emission at the light cylinder (Lyubarski 1996) or in vacuum fields in the magnetosphere (Higgins $\&$ Henriksen 1996). With the larger number of obverved $\gamma$-ray pulsars and the improved sensitivity of the detectors, predictions of the models can now be tested and their parameters more tightly constrained.

\section{Observed Patterns}

There are several patterns and correlations that show up in recent CGRO data which the existing models must be able to explain (see the review by Thompson (1996) for a more complete discussion of the observations). Briefly, these are: 
1)Light curves having double peaks (most widely separated) with bridge emission are common.

2) The spectral hardness shows a systematic variation through the profile that is a maximum in the bridge region (Fierro et al. 1996).

3) The hardness of the phase-averaged spectrum increases with age (i.e. $P / 2 \dot{P}$ ) (Thompson et al. 1994).

4) All pulsars show high energy breaks or cutoffs in their spectrum (Nel \& DeJager 1995).

5) There is a correlation between $\gamma$-ray luminosity and polar-cap current (or open field-line voltage) $\propto P^{-3 / 2} \dot{P}^{1 / 2}$.

Despite these remarkable regularities, there are some puzzling misfits and lack of pattern. Except for the Crab, where there is almost complete alignment of the pulse phase across all wavelengths, there is no alignment of the $\gamma$-ray pulses with the light curves at other wavebands. All of the spectral cutoffs occur above $1 \mathrm{GeV}$, except in PSR1509-58, where the cutoff occurs between 4 and $30 \mathrm{MeV}$. PSR0656+14 is the one exception to the $\gamma$-ray luminosity-polar cap current correlation. Geminga is the only $\gamma$-ray pulsar that is not a radio pulsar.

\section{Polar Cap Models}

Polar cap models divide according to the mechanism of acceleration above the surface of the neutron star (see Usov 1996, for more detailed review of acceleration mechanisms). In pulsars having $\Omega \cdot \mathbf{B}>0$, where $\Omega$ is the direction of the rotation axis, the corotation charge $(-\Omega \cdot B / 2 \pi \epsilon c)$ above the magnetic poles is negative. If the neutron star surface temperature, $T_{s}>T_{e} \simeq 10^{5} \mathrm{~K}$ where $T_{e}$ is the electron surface binding temperature, then electrons are freely released from the surface to be accelerated in parallel electric fields induced by spacecharge-limited flow (Michel 1991), field-line curvature (Arons \& Scharlemann 1979, Arons 1983) and inertial frame dragging (Muslimov \& Tsygan 1992). In these models, $E_{\|}=0$ at the surface and increases with height. In pulsars having $\boldsymbol{\Omega} \cdot \mathbf{B}<0$, the corotation charge above the poles is positive and if $T_{s}<T_{i} \simeq 10^{5}$ $\mathrm{K}$, where $T_{i}$ is the ion surface binding temperature, then the ions are trapped in the surface. Since the corotation charge cannot be supplied, a vacuum gap and thus a non-zero $E_{\|}$grows at the stellar surface (Ruderman \& Sutherland 1975). In most cases, the $E_{\|}$is shorted out at some altitude by the onset of electronpositron pair cascades in the strong magnetic field. However, the creation of bound-pairs (Shabad \& Usov 1985) instead of free-pairs in fields $B \gtrsim 0.1 B_{\text {cr }}$ could delay the shorting out of $E_{\|}$to larger heights above the surface, thus increasing the acceleration energy. But Usov \& Melrose (1995) found this to be an important effect only in Ruderman-Sutherland type models.

Several different kinds of models for polar cap pair cascades have been studied. If the accelerated primary particles reach energies of $\Gamma \geq 10^{6}$, then curvature radiation will dominate their energy loss. For $\Gamma \lesssim 10^{6}$ inverse Compton scattering of X-rays, either non-thermal cascade emission or thermal emission from a hot polar cap, will be more important. More specifically, the energy loss for 


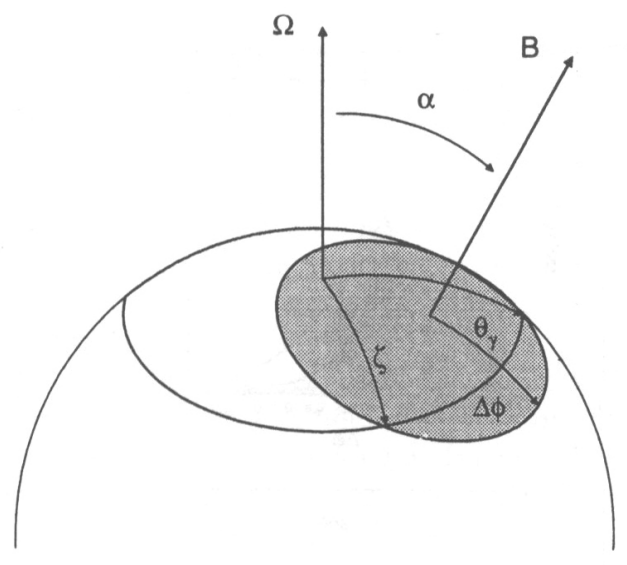

Figure 1. Geometry of single polar cap (SPC) emission. $\zeta$ is the observer polar angle.

curvature radiation of an electron moving parallel to a magnetic field with radius of curvature $\rho_{6} \equiv \rho_{c} / 10^{7} \mathrm{~cm}$ will exceed the energy loss due to non-magnetic inverse Compton scattering of blackbody radiation at temperature $T_{6} \equiv T / 10^{6}$ $\mathrm{K}$, when $\Gamma_{\text {curv }} \gtrsim 2 \times 10^{6} \rho_{7} T_{6}^{2}$ (valid for $\Gamma \gtrsim 10^{4}$ in fields around $10^{12} \mathrm{G}$, Dermer 1990). At $50 \lessgtr \Gamma \lessgtr 10^{4}$, resonant Compton scattering will be important because the soft photons at temperatures near $10^{6} \mathrm{~K}$ will be blueshifted into the cyclotron resonance in the electron rest frame, greatly enhancing the scattering cross section and thus the energy loss rate (Daugherty \& Harding 1989, Dermer 1989). The accelerated particles will radiate $\gamma$-rays by curvature radiation, inverse Compton scattering (or a combination of both) which then pair produce by the process $\gamma \rightarrow e^{+} e^{-}$in the strong magnetic field. The pairs are produced in excited Landau states which decay by emission of synchrotron photons, many of which will also produce pairs. The cascade will continue, reprocessing and thus softening the primary emission spectrum, until the photons escape.

Although it is possible for emission from both polar caps to be visible to observers in the proper orientation, these models have focussed in the last few years on emission from a single polar cap (SPC). In both the curvature radiationinduced pair cascade (CRPC) and the Inverse Compton-induced pair cascade (ICPC) models, the $\gamma$-ray emission pattern is a hollow cone. Since the radius of curvature of the magnetic field lines is infinite at the poles and decreases towards the polar cap rim, the curvature emission is a maximum at the rim in the CRPC models. In the ICPC models, the emission is also maximum at the rim where the angle between the relativistic particles moving along field lines and the soft photons is largest. The $\gamma$-ray beam half-angle, $\theta_{\gamma}$, is determined approximately by the locus of the tangents to the outermost open field lines:

$$
\tan \theta_{\gamma} \simeq \frac{3 \theta_{p c}\left(1-\theta_{p c}^{2} \frac{r}{R}\right)^{1 / 2}\left(\frac{r}{R}\right)^{1 / 2}}{3\left(1-\theta_{p c}^{2} \frac{r}{R}\right)-1}
$$



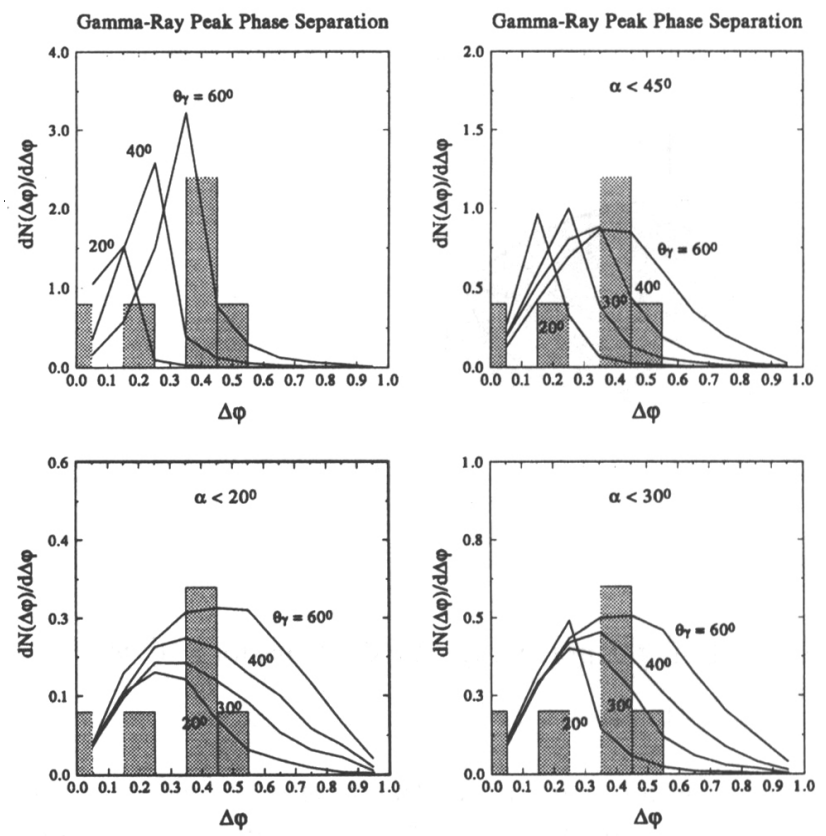

Figure 2. Simulated (solid lines) and observed (shaded histogram) distributions of pulse peak separation, for uniform (upper left) and limited obliquity $\alpha$.

where $\theta_{p c} \ll 1$ is the polar cap half-angle, $r$ is the radius of emission and $R$ is the neutron star radius. When $\theta_{\gamma} \sim \alpha$ as shown in Figure 1, an observer may see a broad double-peaked $\gamma$-ray pulse profile, with bridge emission from inside the polar cap rim. In CRPC models one can allow for extended acceleration regions (up to several stellar radii) above the polar cap, and the outward flaring of the field lines with height rapidly increases $\theta_{\gamma}$ to as large as $30^{\circ}$ to $40^{\circ}$ (Daugherty \& Harding 1996, hereafter DH96). Thus, CRPC models no longer require very small inclinations to accomodate large phase separations in doubly-peaked profiles, as in previous SPC models (Daugherty \& Harding 1994; Sturner et al. 1995, Sturner et al. 1995). Figure 2 shows simulated distibutions of double pulse peak separations, $\Delta \phi$, expected for random observers and various assumed distributions of pulsar obliquity. For uniform $\alpha$, the distributions predict enough large phase separations only for very large $\theta_{i}$, and are then ton sharply peaked to allow many small values of $\Delta \phi$. But when $\alpha$ is limited to even moderately small values, the predicted distributions are more consistent with those observed and will allow smaller values of $\theta_{\boldsymbol{\gamma}}$.

\subsection{Curvature radiation induced pair cascades}

The $\gamma$-ray emission from pair cascades induced by curvature radiation of accelerated particles has been investigated by Daugherty \& Harding (1982, 1994, 
1996) and Chiang \& Romani (1992), assuming a space-charge limited electron a.cceleration model (e.g. Arons 1983) is operating. DH96 found that if the primary electron acceleration is assumed to occur over several stellar radii, then the cascade $\gamma$-ray emission shows agreement with several key features of the observed emission, including the double-peaked light curves with bridge emission (as discussed above) as well as phase-averaged and phase-resolved spectra. In particular, the CRPC models predict high-energy spectral cutoffs, due to magnetic pair production, at several $\mathrm{GeV}$. The pair production attenuation cutoffs are much sharper than exponential cutoffs due to a cutoff in the particle distribution, and can fit the very steep cutoffs in observed $\gamma$-ray pulsar spectra. Some correlation of surface magnetic field with pair production or photon splitting (in the case of PSR1509-58, see Harding \& Baring 1996) cutoff energy is predicted, with higher $B$ producing lower cutoff energies. The CRPC models can account for the systematic soft-hard-soft hardness variation of the pulse profile of Vela. (Kanbach et al. 1994). The hardest emission is predicted to occur near the pole, where the emission is pure curvature radiation that is not softened by cascading. Near the polar cap rim, where the peak emission originates, the cascades are most extended, producing a softer spectrum with a lower cutoff energy. The emission outside the rim, and outside the peaks in the pulse profile, comes from high-altitude curvature radiation from primary particles that have lost most of their energy and has the lowest cutoff, but not as sharp as that of the peak spectra. Very importantly, the CRPC/SPC model predicts pulsed emission at all phases, as has been observed (Fierro et al. 1996).

The observed increase in hardness of the phase-averaged spectrum with age can also be understood in the CRPC model. The hardness of a CRPC cascade spectrum primarily depends on the number of photon generations in the cascade. As noted by Lu et al. (1994), there is a rough correlation between the spectral indices of the observed $\gamma$-ray pulsars and a theoretical estimate of the number of polar cap cascade generations. The primary curvature radiation spectrum has a photon index of $5 / 3$ if the particles have significant energy loss and $2 / 3$ if they do not. Significant energy loss of the primary particles will occur for short period $\left(P<P_{c} \simeq 0.1\right)$ pulsars, where the polar cap half-angle, $\theta_{p c} \propto P^{1 / 2}$, is larger and thus the field line radius of curvature at the polar cap rim, is smaller. The input curvature spectrum is then softened on each generation of pair production and synchrotron emission. The spectral index of the escaping $\gamma$-ray spectrum will be (Harding \& Daugherty 1996)

$$
\gamma(\xi)=2-\frac{1}{2^{\xi-1}}\left(2-\gamma_{1}\right) \stackrel{\xi \rightarrow \infty}{\longrightarrow} 2
$$

where the number of cascade generations, $\xi$, is allowed to be a non-integer and $\gamma_{1}$ is the spectral index of the primary curvature spectrum. The number of generations will generally increase with decreasing pulsar period and increasing surface magnetic field strength $B_{\mathrm{o}}$, because the mean free path for pair production will decrease. The generation number will also decrease with the height above the surface at which the bulk of the cascade occurs. Figure 3 shows the predicted dependence of spectral index with the parameter $P / B_{12}$ for different radii $r$ of the cascade. Since the pulsar characteristic age $\tau \propto\left(P / B_{12}\right)^{2}$, a correlation of spectral index with age is predicted. Figure 3 also shows a comparison of predicted and observed $\gamma$ for the.observed $\gamma$-ray pulsars. Several pulsars with ages 


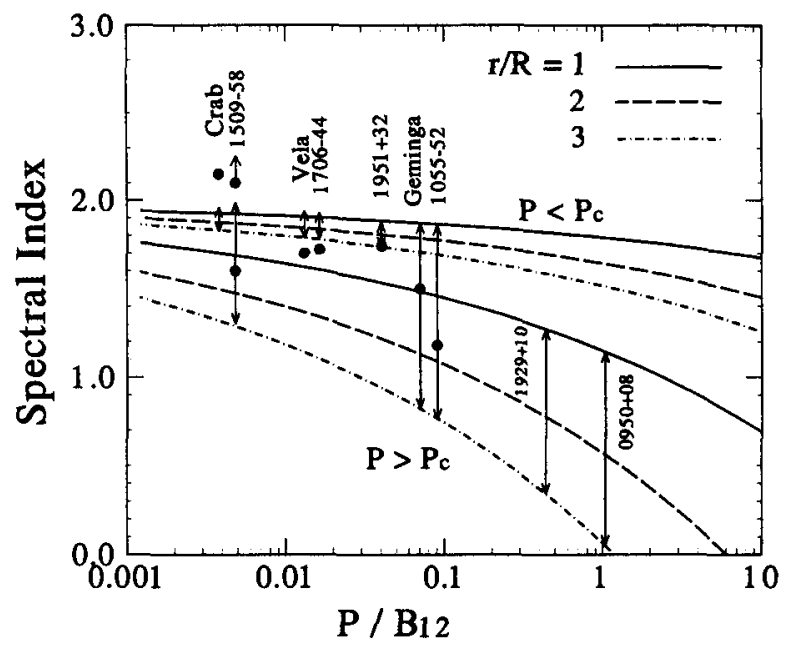

Figure 3. Predicted $\gamma$-ray spectral index vs. $P / B_{12}$.

larger than $10^{6} \mathrm{yr}$ and low magnetic fields, PSR1929+10 and PSR0950+08, that should be detectable by EGRET based on their predicted fluxes, have extremely low predicted spectral indices, indicating that the bulk of their $\gamma$-ray emission could lie above the EGRET range.

\subsection{Inverse-Compton induced pair cascades}

When $\Gamma \lesssim \Gamma_{\text {curv }}$, inverse Compton emission will provide the primary spectrum to initiate the cascade, which then proceeds via one-photon pair production and synchrotron radiation, similar to the curvature initiated cascades. ICPC models have been investigated by Dermer \& Sturner (1994) and by Sturner et al. (1995). The energy to which the primary electrons are accelerated is assumed to be that only of the space-charge limited flow potential, $\Gamma_{\mathrm{SD}} \approx 10^{6} B_{12}^{1 / 2} / P$. However, the energies required to radiate photons with energies up to several $\mathrm{GeV}$ with Compton scattering are much lower than for primary curvature radiation. The final $\gamma$-ray spectra have power law indices that may be as hard as $\gamma=0$ in the case of no cascading to $\gamma \simeq 2$ with a synchrotron photon cascade contribution. Since pulsars with larger $\Gamma$ will have softer spectra, this model may also explain the correlation of spectral hardness with apparent age.

ICPC models cannot produce cascades several stellar radii above the neutron star surface in pulsars whose thermal X-ray emission from a hot polar cap dominates the non-thermal X-rays, because the flux of soft photons decreases rapidly with altitude. In order to reproduce the widely separated double-peaked profiles, these models must require very small obliquities, $\alpha \sim \theta_{\gamma} \leqslant 5^{0}$ (Sturner \& Dermer 1995). 


\section{Outer Gap Models}

Vacuum gaps may grow in the outer magnetosphere along null charge surfaces, which separate regions of the opposite sign of the corotation charge. Along open field lines threading this surface, charges of one sign will escape through the light cylinder and cannot be replaced, because charge coming from the polar cap below has the opposite sign. Particles can be accelerated by the resulting $E_{\|}$in the gap to energies limited by curvature radiation reaction (Cheng, Ho \& Ruderman 1986). In outer gap models, the spectral cutoffs are due to this limit on the primary particle energy and are predicted to be somewhat more gradual than the attenuation cutoffs of polar cap models. Unlike the case in polar cap models, the magnetic fields in the outer gap are too weak for one-photon pair production to play a significant role in the cascade. Instead, the curvature photons undergo photon-photon pair production with thermal X-rays from the polar caps (as in the case of Vela or Geminga) or non-thermal X-rays produced by the cascade (as in the Crab). In the Crab pulsar, the $\gamma$-ray emission is produced by synchrotron self-Compton radiation from the pairs, with the lower energy part of the spectrum dominated by the synchrotron component and the higher energy spectrum dominated by inverse Compton (Ho 1989). In Vela-like pulsars with periods around $0.1 \mathrm{~s}$, a large obliquity is required to bring the inner gap radius closer to the neutron star. This has the dual purpose of increasing the optical depth to pair production with thermal X-ray photons (Romani 1996) and increasing the gap magnetic field, which moves the "death" line to longer periods (Chen \& Ruderman 1993). The spectra of these pulsars will be primarily curvature radiation and it is possible to reproduce the observed soft-hard-soft variation through the profile (Romani 1996).

The $\gamma$-ray pulse profiles from the outer gaps have been investigated, in a simple geometrical model, by Chiang \& Romani (1994), who assumed that the emission from gap accelerated particles is beamed along magnetic field lines and also assumed a spatial and energy dependence of the radiation. They were able to produce double peaked profiles with bridge emission separated by $140^{\circ}$, similar to the Crab and Vela pulsars. The $\gamma$-rays from the outer gap are emitted into fan beams with large solid angle $\Omega_{\gamma} \sim 1$ sr.

\section{Conclusions}

Polar cap and outer gap models have both evolved in order to account for the observed emission features. Polar cap models now assume that emission originates from only one magnetic pole and at higher altitudes. Outer gap models must allow the gaps to extend closer to the neutron star surface. The $\gamma$-ray emission must be either a hollow cone (CRPC and ICPC polar cap models) or a curved fan beam (outer gap models) to produce double-peaked profiles, bridge emission and the soft-hard-soft hardness variation; however ICPC models do not predict emission outside the pulses. CRPC models predicted high-energy spectral cutoffs (Daugherty \& Harding 1982) and a correlation of $\gamma$-ray luminosity with polar-cap current (Harding 1981), both of which have been observed by CGRO. Outer gap models can also produce spectra turnovers, but à luminosity-current correlation is not predicted. Polar cap models predict an increase in spectral 
hardness with age; outer gap models may predict this as well but it has not been quantified. Outer gap models can more naturally account for phase differences between $\gamma$-ray and lower energy pulses if the lower energy is assumed to come from near the surface. It is quite possible that elements of all these models (and more) will be necessary to account for the diverse features of $\gamma$-ray pulsars.

\section{References}

Arons, J. 1983, ApJ, 266, 215.

Arons, J. \& Scharlemann, E.T. 1979, ApJ, 231, 854.

Chen, K. \& Ruderman, M. A. 1993, ApJ, 402, 264.

Cheng, K. S., Ho, C. and Ruderman, M. A. 1986, ApJ, 300, 500.

Chiang, J. \& Romani, R. W. 1992, ApJ, 400, 629.

Chiang, J. \& Romani, R. W. 1994, ApJ, 436, 754.

Daugherty, J. K. and Harding, A. K. 1982, ApJ, 252, 337.

Daugherty, J. K. and Harding, A. K. 1989, ApJ, 336, 861.

Daugherty, J. K. \& Harding A. K. 1994, ApJ, 429, 325.

Daugherty, J. K. \& Harding A. K. 1996, ApJ, 458, 278.

Dermer, C. D. 1989, ApJ, 347, L13.

Dermer, C. D. 1990, ApJ, 360, 197.

Dermer, C. D. \& Sturner, S. J. 1994, ApJ, 420, L75.

Fierro, J. M. 1996, Ph.D. Thesis, Stanford University.

Harding, A. K. 1981, ApJ, 245, 267.

Harding, A. K. \& Baring, M. G. 1996, these proceedings.

Harding, A. K. \& Daugherty, J. K. 1996, in preparation.

Higgins, M. G. \& Henriksen, R. N. 1996, MNRAS, submitted.

Ho, C. 1989, ApJ, 342, 396.

Kanbach, G. et al. 1994, A\&A, 289, 855.

Lu, T., Wei, D. M. \& Song, L. M. 1994, A\&A, 290, 815.

Lyubarskii, Y. E. 1996, A\&A, in press.

Michel, F. C. 1991, Theory of Neutron Star Magnetospheres, Univ. of Chicago Press.

Muslimov, A. G. \& Tsygan, A. I. 1992, MNRAS, 255, 61.

Nel, H. I. \& De Jager, O. C. 1995, Ap\&SS, 230, 299.

Romani, R. W. 1996, preprint.

Romani, R. W. \& Yadigaroglu, I.-A. 1994, ApJ, 438, 314.

Ruderman, M. A. and Sutherland, P. G. 1975, ApJ, 196,51.

Shabad \& Usov 1985, Ap\&SS, 117, 309.

Sturner, S. J. \& Dermer, C. D. 1994, ApJ, 420, L79.

Sturner, S. J. \& Dermer, C. D. 1995, ApJ, 461, 872.

Sturner, S. J., Dermer, C. D. \& Michel, F. C. 1995, Ap.J, 445, 736.

Thompson, D. J. et al. 1994, ApJ, 436, 229.

Thompson, D. J. 1996, these proceedings.

Usov, V. V. 1996, these proceedings.

Usov, V. V. \& Melrose, D. B. 1995, Aust. J. Phys., 48, 571. 